\title{
From lifetime to evolution: timescales of human gut microbiota adaptation
}

\section{Sara Quercia ${ }^{1}$, Marco Candela ${ }^{1}$, Cristina Giuliani ${ }^{2}$, Silvia Turroni ${ }^{1}$, Donata Luiselli ${ }^{2}$, Simone Rampelli ${ }^{1}$, Patrizia Brigidi ${ }^{1}$, Claudio Franceschi ${ }^{3,4,5,6,7}$, Maria Giulia Bacalini ${ }^{3,4}$, Paolo Garagnani ${ }^{3,4,8}$ and Chiara Pirazzini ${ }^{3,4}$ *}

${ }^{1}$ Department of Pharmacy and Biotechnology, University of Bologna, Bologna, Italy

${ }^{2}$ BiGEA, Department of Biological, Geological and Environmental Sciences, Laboratory of Molecular Anthropology \& Centre for Genome Biology, University of Bologna, Bologna, Italy

${ }^{3}$ DIMES, Department of Experimental, Diagnostic and Specialty Medicine, University of Bologna, Bologna, Italy

${ }^{4} \mathrm{ClG}$, Interdepartmental Centre "L. Galvani" CIG, University of Bologna, Bologna, Italy

${ }^{5}$ IRCSS, Institute of Neurological Sciences of Bologna, Bologna, Italy

${ }^{6}$ IGM-CNR, Institute of Molecular Genetics, Unit of Bologna IOR, Bologna, Italy

${ }^{7}$ CNR, Institute of Organic Synthesis and Photoreactivity (ISOF), Bologna, Italy

${ }^{8}$ CRBA, Center for Applied Biomedical Research, St. Orsola-Malpighi University Hospital, Bologna, Italy

\section{Edited by:}

Anton G. Kutikhin, Research Institute for Complex Issues of Cardiovascular Diseases Under the Siberian Branch of the Russian Academy of Medical Sciences, Russia

\section{Reviewed by:}

Carl James Yeoman, Montana State University, USA

Valerio lebba, 'Sapienza' University of Rome, Italy

Riccardo Calvani, Catholic University of the Sacred Heart School of

Medicine, Italy

Anton G. Kutikhin, Research Institute for Complex Issues of Cardiovascular Diseases Under the Siberian Branch of the Russian Academy of Medical Sciences, Russia

Arseniy E. Yuzhalin, University of Oxford, UK

\section{*Correspondence:}

Chiara Pirazzini, DIMES, Department

of Experimental, Diagnostic and Specialty Medicine, University of

Bologna, Via San Giacomo,

1240126 Bologna, Italy

e-mail: chiara.pirazzini5@unibo.it
Human beings harbor gut microbial communities that are essential to preserve human health. Molded by the human genome, the gut microbiota (GM) is an adaptive component of the human superorganisms that allows host adaptation at different timescales, optimizing host physiology from daily life to lifespan scales and human evolutionary history. The GM continuously changes from birth up to the most extreme limits of human life, reconfiguring its metagenomic layout in response to daily variations in diet or specific host physiological and immunological needs at different ages. On the other hand, the microbiota plasticity was strategic to face changes in lifestyle and dietary habits along the course of the recent evolutionary history, that has driven the passage from Paleolithic hunter-gathering societies to Neolithic agricultural farmers to modern Westernized societies.

Keywords: gut microbiota, aging, environmental stimuli, co-evolution, biological adaptation

\section{INTESTINAL MICROBIOTA, STRUCTURE, AND ROLE IN HUMAN PHYSIOLOGY}

Human beings co-evolved as superorganisms as the result of the mutualistic relationship with the enormous microbial community that resides in human gastrointestinal tract (GIT); this ecosystem is better known as gut microbiota (GM; Turnbaugh et al., 2007). The GM reaches the highest cell concentration in the colon, with a density of $10^{12} \mathrm{CFU} / \mathrm{g}$ of intestinal content and represents the most densely populated and biodiverse ecosystem on earth (O'Hara and Shanahan, 2006; Ley et al., 2008a). The GM presents a very particular phylogenetic structure, resulting in a sparsely branched tree, with a high degree of radiation at the ends. Indeed, out of the 100 different bacterial phyla detected on our planet, only seven are found in our gut - Firmicutes, Bacteroidetes, Actinobacteria, Proteobacteria, Verrucomicrobia, Tenericutes, and Fusobacteria - of which Firmicutes and Bacteroidetes represent together up to 90\% of the ecosystem (Costello et al., 2009). Conversely, the GM shows an impressive biodiversity at lower phylogenetic levels with up to 1000 different species being detected by next generation sequencing-based approaches (Qin et al., 2010). Interestingly, the species-level GM composition varies dramatically among people, and each subject owns a very unique subset of microorganisms, that consists of hundreds of the more than 1000 species detected in the GM of the entire human population. The total genome of these microorganisms, often referred to as the intestinal microbiome, has been estimated to contain 150 times more genes than the human one, providing the host with essential functional traits that human beings have not evolved on their own (Qin et al., 2010). For instance, the carbohydrate-active enzymes encoded in the microbial glycobiome allow the host to extract energy from otherwise 
indigestible polysaccharides (Gill et al., 2006), complementing the poor human glycobiome diversity. Indeed, the functional assignment of the gut microbiome revealed an extraordinary diversity of gene clusters involved in carbohydrate metabolism (Koropatkin et al., 2012). Moreover, the gut microbiome is enriched in genes involved in the production of vitamins, cofactors, and secondary metabolites, further supporting its important role in host nutrition (Bäckhed etal., 2004; Clemente et al., 2012). The GM is also an active component of the human immune system (Round and Mazmanian, 2009; Maynard et al., 2012). The cross-talk with intestinal microbes has been shown to be essential for the maturation of a correct immune function in early life and to preserve a well-balanced immune homeostasis later in life (Kamada et al., 2013). Finally, a new and only sparsely explored role of the GM in human physiology is its potential to modulate the function of the gut-brain axis. Indeed, accumulating data from studies carried out in mouse models suggest how the GM and its metabolites could affect the behavior and pain, in addition to depression, anxiety and other disorders belonging to the central nervous system (Cryan and Dinan, 2012). Gut commensals are capable of activating neural pathways and modulating signaling to the central nervous system through their metabolite production (Foster and McVey Neufeld, 2013). In particular, studies carried out on mice demonstrated a strategic role for commensal bacteria in programming the hypothalamic-pituitary-adrenal (HPA) stress responsiveness at early developmental stages, when brain plasticity is still preserved (Sudo et al., 2004). Indeed, germ-free mice showed an enhanced HPA response to restraint stress, which was reversed by their re-conventionalization at early stages of development. According to the authors, both a cytokine-mediated humoral route and a neural mediated pathway could be involved in the microbiota modulation of the endocrine response in early life. Moreover, as observed by Diaz Heijtz et al. (2011) in mice, the GM can also affect the synaptogenesis during the perinatal period.

The recent use of germ-free and gnotobiotic mice has allowed the field to disentangle the complexity of the GM-host transgenomic metabolism, shedding light on the specific role of GM metabolites in host physiology (Nicholson et al., 2012). In particular, the endpoints of the GM polysaccharide fermentation, short-chain fatty acids (SCFAs) - most abundantly acetate, propionate, and butyrate - are key GM metabolites, with a multifactorial role in human health and homeostasis. These acids have been shown to play a pivotal role in host nutrition and energy homeostasis, controlling energy production, and storage as well as the appetite. Butyrate represents the main energetic substrate for the colonic epithelial cells (Russell et al., 2013) and both butyrate and propionate have been reported to activate intestinal gluconeogenesis (IGN) through two complementary mechanisms: butyrate triggers IGN gene expression in the gut via cAMP-dependent mechanisms, whereas propionate activates IGN by gene expression through gut-brain neural circuits and itself represents a substrate for IGN (De Vadder et al., 2014). According to the authors, this last propionate-dependent mechanism of IGN induction has been defined as strategic to provide the host with several metabolic benefits on body weight and glucose control. Besides the nutritional role, SCFAs have been reported to be involved in the maintenance of immune homeostasis (Arpaia et al., 2013). Through their production the GM controls the epithelium inflammation rate and drives the production and migration of specific immunological cells. Effectively, propionate governs the de novo peripheral regulatory $\mathrm{T}$ cell (Treg) generation and, together with acetate, drives their homing in the colon. Furthermore, propionate has been involved in the enhancement of hematopoiesis of dendritic cells with an impaired Th2 activation (Trompette et al., 2014). On the other hand, butyrate has the ability to regulate the production of pro-inflammatory cytokines (Segain et al., 2000), exerting a local immunomodulatory activity. Moreover, it is involved in the extrathymic Treg generation. In addition, the protective activity exerted by butyrate on the gut epithelium has been reported, as it stimulates the release of mucins (Petersson et al., 2011).

\section{GENETICS OF HOST AND MICROBIOTA}

Host genetics and the GM are linked together by an intense crosstalk and this interaction is dynamic throughout the course of our life. Several studies have been conducted to determine the impact of host genetics on the GM composition providing conflicting results. To address how the host genotype and the environment influence the GM composition, a study on the fecal microbiota of monozygotic and dizygotic twin pairs concordant for leanness or obesity, and their mothers was conducted (Turnbaugh et al., 2009). The authors found that the GM of monozygotic twin pairs had a degree of similarity that was comparable to that of dizygotic twin pairs, highlighting that the environment impacts the GM composition more than the genetics does. It was also reported that family members harbor a similar GM composition and share a "core microbiome" made of several microbial genes. However, a study conducted on related and unrelated children reported that the highest level of similarity was found in genetically identical twins (Stewart et al., 2005).

Several studies reported that single host genes, i.e., MEditerranean FeVer, APOA1, NOD2, and FUT2 affect the GM by altering its composition or by reducing the degree of bacterial diversity (Khachatryan etal., 2008; Petnicki-Ocwieja et al., 2009; Zhang et al., 2010; Frank et al., 2011; Wacklin et al., 2014).

Murine models proved to be very useful to clarify the effect of genetics on the GM. One of the first studies focused on the interaction host genetics-GM was based on observations on the GM reconstruction process occurring after a course of antibiotics (Vaahtovuo et al., 2003). It was observed that the colonization of the GM depends on the genotype of the host, and differences in communities between mouse strains were observed, supporting the idea that the gut community is not established by chance but is influenced by the host genetic background. Kovacs et al. (2011) studied particular recombinant inbred mouse strains to assess the relative role of the host genotype in the GM composition and they reported that the mouse genetic background is a strong determinant in shaping the intestinal microbiota. To address how environmental factors and host genetic factors combine to shape the GM, Benson et al. (2010) explored the associations between host quantitative trait loci (QTL) and the GM composition in mice. Eighteen host's QTL showing a significant association with the relative abundance of specific microbial taxa were identified. 
Even if litter and cohort effects accounted for some of theGM variation, according to the authors host genetics had a greater impact on the GM variability.

All these studies provide information that supports the idea that the host genetics and the GM interact with each other deeply, and we can speculate that changes in the GM composition could boost the different genetic make up of every individual.

\section{MICROBIOTA ADAPTATION TO DAILY LIFE: MICROBIOME PLASTICITY IN RESPONSE TO DIFFERENT DIETS AND HOST PHYSIOLOGY}

The human GM is a complex dynamic system with the potential for multistability. Indeed, Faith et al. (2013) found that on average $40 \%$ of the microbial strains harbored in an adult's intestine was variable in a 5-year sampling period. In a mutualistic context the GM makes sudden jumps from different steady states under the pressure of environmental and endogenous factors, such as diet, age, host genetics, and physiological state (Candela et al., 2012).

The most rapid observable response of the gut microbial community is the reaction to daily dietary changes. Through a study where high-fat low-fiber and low-fat high-fiber diets were compared, changes in the microbiome composition were detected within $24 \mathrm{~h}$ of controlled feeding, confirming that the human gut ecosystem plasticity can respond efficiently and rapidly to external variables (Walker et al., 2011). However, as reported by Wu et al. (2011), short- and long-term dietary interventions differently impact the GM composition. Effectively, some bacterial groups are more likely to be influenced only by short-term dietary intervention, while others, namely those referred to as human enterotypes (Arumugam et al., 2011), are affected only by long-term intervention.

The correlation between nutrients and the GM composition was investigated in a caloric restriction study realized in 18 lean subjects over a 4 -day period. The outcomes showed that nutritional compounds, like proteins and fibers, affect the phylogenetic and functional structure of the gut microbial community (Muegge et al., 2011). The connection between the GM phylogenetic profile and the ingestion of a specific nutrient, namely fermentable carbohydrate, was also observed in a recent study conducted in 14 overweight men in a 3-week period of intervention (Walker et al., 2011).

It has been recently demonstrated that animal-based and plantbased diets deeply impact on the GM (David et al., 2014). Both diets were administered for 5 days to 10 young US adults and the microbial community composition, metabolic products, and gene expression were analyzed. Interestingly, dietary changes to the plant- or animal-based diet resulted in marked microbiota changes only 1 day after the diet modification. In particular, the plant-based diet was associated with the presence of fibrolytic SCFA producers as Roseburia, Eubacterium rectale, and Faecalibacterium prausnitzii, while the animal-based diet resulted in the increase of potentially putrefactive microorganisms, such as Bacteroides and the bile tolerant Bilophila wadsworthia and Alistipes. It was observed that the animal-based diet had a greater impact on the GM structural and functional layout than the only plantbased diet. Lower levels of metabolic products resulting from the fermentation of carbohydrates and greater levels of the products resulting from the fermentation of amino acids were reported in individuals with the animal-based diet. Finally, the animalbased diet was associated with an increased expression of genes involved in the biosynthesis of vitamins and genes involved in the metabolism of products resulting from the consumption of meat (David et al., 2014). Interestingly, besides dietary substrates, the GM also relies on host-derived glycans secreted in the mucus as a nutrient source in the gut (Kashyap et al., 2013). Indeed, genetically dictated changes in host mucus glycan composition, such as the presence or absence of terminal fucose residues, have been shown to significantly impact the GM structure and function. This provides a global view where the diet and the host genotype interact to modulate the GM configuration.

The ability of the GM to re-program itself in response to different stimuli is necessary to adapt to the metabolic requirements of the host corresponding to different physiological states. For instance, pregnancy represents a period of deep physiological changes during which the GM composition adjusts according to the growth of the fetus and the lactation period. According to Koren et al. (2012), pregnancy is characterized by a greater inflammation tone, reduced insulin sensitivity, and body fat increase.

These traits are supported by a pregnancy-associated GM profile, whose main features are the expansion of Proteobacteria and Actinobacteria and a decrease in richness. Interestingly, such modifications in the GM composition persist for 1 month after birth and afterward the adult-like microbiota configuration is restored.

While the GM virtually varies in response to any changes in environmental and endogenous factors, the GM adaptation to extreme conditions - as an abnormal dietary sugar and fat intake or chronic inflammation - breaks the microbiota-host mutualistic homeostasis, lowering the ecosystem diversity, and overcoming the resilience of the microbiota-host symbiosis. The microbiota observed in obese people represents an appropriate example of unbalanced GM configuration driven by the Westernized diet and lifestyle (Ridaura et al., 2013). The functional annotation of the obese-type gut microbiome revealed a decreased functional diversity and an enrichment in genes involved in carbohydrate, lipid, and amino acid metabolism, showing an overall increased fermentative capacity with respect to the lean-type microbiome (Turnbaugh et al., 2009). Moreover, very recently, Schulz et al. (2014) demonstrated that a high-fat diet mediates shifts in the GM composition that promote intestinal carcinogenesis by compromising the Paneth-cell-mediated antimicrobial host defenses. On the other hand, inflammatory bowel disease (IBD) is a paradigmatic model to elucidate the self-sustained inflammatory loop that is established in the gut as a result of an inflammationinduced microbiota dysbiosis. In particular, inflammation forces GM to change toward a pro-inflammatory pathobionts-enriched profile, which consolidates the host inflammatory tone (Round and Mazmanian, 2009).

Therefore, the role of the GM as a plastic factor in response to environmental or endogenous stress is essential for the maintenance of the mutualistic relationship with the host. But under some specific circumstances the microbial community can be 
forced to shift to a disease-associated configuration with the breaking of the homeostasis balance.

\section{MICROBIOTA ADAPTATION TO DIFFERENT AGES}

The human GM describes an evolutionary trajectory along the course of human life. The GM ecosystem changes its structural and functional layout from early infancy to old age, providing the host with ecosystem services finely calibrated for each stage of life (Yatsunenko et al., 2012). For instance, the peculiar GM composition during infancy exerts specific functions for the infant biology, supporting the immune system education, brain development, and host nutrition (Candela et al., 2013). At weaning, the GM gains diversity and develops new physiological functions, in order to fulfill the adult age-related requirements, such as the need to extract energy from the variable array of complex polysaccharides characterizing the adult diet.

The individual microbial layout begins to be formed immediately during delivery (Jost et al., 2012). We are born sterile and environmental microbes immediately colonize us (Palmer et al., 2007). The infant' GIT is firstly colonized, just a few hours after birth, by facultative anaerobic bacteria, i.e., enterobacteria, staphylococci, and streptococci. Over time, the decreased amount of available oxygen allows strictly anaerobic bacteria to settle in the intestine, modifying the intestinal environment (Vael and Desager, 2009). In particular, Jost et al. (2012) analyzed the bacterial composition in feces from seven healthy vaginally delivered, breast-fed neonates at different times after birth. They observed that, during the first days of life, anaerobes, i.e., Bifidobacterium and Bacteroides, outnumbered facultative anaerobes in all seven neonates, pointing out that anaerobes may become dominant early in life and that the switch from facultative to strict anaerobes may occur at a very early stage. The infant-type microbiota is thus characterized by the dominance of Bifidobacterium and the presence of Staphylococcus, Streptococcus, and Enterobacteriaceae as other major components. With a relatively low degree of diversity, the infant-type GM is capable of tremendous fluctuations over time, with an individual-specific temporal pattern of variation in species composition (Palmer et al., 2007; Jost et al., 2012). The delivery mode is one of the factors that most influence early infants' microbiota composition (Dominguez-Bello et al., 2010). Indeed, the authors observed that the vaginally delivered infants acquired bacterial communities resembling their own mother's vaginal microbiota, while the cesarean section infants transiently harbored bacterial communities similar to those found on the mothers' skin surface.

Largely dominated by Bifidobacterium and Enterobacteriaceae with an extraordinary rate of variation over time, the infanttype microbiota is functionally structured to educate the infant immune system through an intense, yet controlled, immunological dialog. Centanni et al. (2013) demonstrated that in infants the phylogenetic structure of the enterocyte-associated GM fraction was unaffected by the host inflammatory stimulus, probably because the GM of infants is specifically shaped to cope with the dynamic and intense cross-talk with the host immune system that is necessary for immune education. Recently, it has been shown that the diversity in the GM composition in infants is more important than the prevalence of specific bacterial taxa in the determination of the risk of immunological diseases later in life, i.e., allergic disease and asthma (Bisgaard et al., 2011; Abrahamsson et al., 2013). Furthermore, the infant GM also responds to precise developmental and nutritional needs crucial for the infant, such as the development and functionality of the central nervous system (Sudo et al., 2004; Collins et al., 2012), as well as the specific vitamin requests (Yatsunenko et al., 2012). Recently, fascinating hypotheses extending the GM-dependent immune and metabolic programming to the perinatal period have been advanced (Rautava et al., 2012). However, until confirmed by robust experimental findings, such hypotheses need to be taken with caution (Hanage, 2014).

The infant-type GM is subject to profound fluctuations until weaning when, with the introduction of solid food, it shifts toward the adult-type microbiota, with the progressive acquisition of taxonomic and functional complexity, such as a wide array of carbohydrate-active enzymes. This shift results in a profound change in the GM composition that goes from a bifidobacteriaenriched community to another one dominated by Firmicutes and Bacteroidetes, resembling more and more the microbiota of an adult, characterized by increased functionality and stability (Koenig etal., 2011). This adult-type microbiota is functionally structured to metabolize the whole complexity of the plant polysaccharides contained in the adult diet and provides mutual benefits to the host (Vanhoutte et al., 2004). Indeed, the microbiota takes advantage of a warm and nutrient-rich environment in which it can settle, while the host can benefit from an easyfitting metabolic equipment that can provide essential factors and increase the host's digestive capacity (Lozupone et al., 2012). A strong selection toward a readily changeable individual microbiome profile has been shown (Candela et al., 2012). This is the consequence of the inherent degree of plasticity of this bacterial ecosystem in adults, which allows the GM to change in response to environmental/endogenous factors, and the uniqueness of our physiology, lifestyle and history (Costello et al., 2012). These result in a peculiar temporal dynamics of the individual GM, always providing an adaptive response to ensure ecosystem services in the face of personalized physiology, immune system, environmental, or dietary exposure and lifestyle (Candela et al., 2013).

With aging and the onset of pathophysiological conditions (e.g., colon cancer, IBD, obesity, type 2 diabetes, and cardiovascular diseases) the GM-host mutualistic relationship progressively becomes compromised (Biagi et al., 2013). In elderly people diet and lifestyle undergo profound variations that include alterations of taste and smell, of gastrointestinal motility, and mastication, resulting in a nutritionally imbalanced diet (Biagi et al., 2010, 2013; Claesson et al., 2011; Drago et al., 2012). These age-related modifications, together with immunosenescence, affect the phylogenetic and functional structure of the gut ecosystem, leading to a microbial composition that favours the bloom of pathobionts (Enterobacteriaceae) to the detriment of immunomodulatory groups (Clostridium cluster IV and XIVa, Bifidobacterium). This age-associated configuration together with the "inflammaging" process could contribute to the creation of a self-sustained proinflammatory loop that is prejudicial for host health (Franceschi et al., 2000a,b; Grignolio et al., 2014). Interestingly, the GM of 
the elderly displays a restricted stability and extreme variability. Recently, a functional description of the aged GM was reported (Rampelli etal., 2013). By using Illumina shotgun sequencing, three centenarians' fecal samples were analyzed and a shift from a saccharolytic to a putrefactive metabolism was reported. Indeed, an increase in the proteolytic potential, a reduction of genes involved in the metabolism of carbohydrates and a reduction of genes involved in SCFA production were observed. These modifications are in agreement with the age-related enrichment of genes belonging to pathobionts, and the authors hypothesized the existence of a pro-inflammatory loop in which pathobionts actively promote the worsening of health status with age. They also speculate that in centenarians some readjustments could occur to counteract the detrimental effects of pathobiont accumulation.

\section{MICROBIOTA ADAPTATION DURING DIETARY SHIFT IN HUMAN EVOLUTION}

Bacteria are part of the evolutionary history of complex organisms and they occupy every ecological niche of our planet. The human GM is the biggest stable symbiont of our body (Costello et al., 2009) and it is characterized by a long adaptive history.

Modern humans, when have moved out of Africa, had to face different environmental challenges (such as food availability, climate changes, and pathogen loads). The main change in the host-microbiota symbiosis likely occurred almost 10,000 years ago, during the Neolithic revolution, also called "agricultural revolution" (De Filippo etal., 2010; Ottaviani et al., 2011). This revolution is based on the transition from hunting and gathering to agriculture and permanent settlements. In this period, the agriculture and animal husbandry have led to natural changes of human lifestyle and shaped modern human genomes. Given its high plasticity, the GM is able to change its composition and to adapt itself, according to diet/food availability, and the advent of agricultural societies could have favored microbial communities able to ferment complex substrates like polysaccharides (Hehemann et al., 2010). However, to date, little is known regarding how the GM has changed during human evolution. One of the most constraining aspects in this research field is the impossibility of having suitable fossil record. Indeed, the study of changes in the GM in human history is complicated by the difficulty in finding well-preserved samples of feces or intestinal samples of different periods (Walter and Ley, 2011). Nevertheless, researchers are developing methods to overcome this limitation. In a very recent paper, Sistiaga et al. (2014) applied gas-chromatographymass spectrometry to Neanderthal's fecal matter to evaluate sterol and stanol level. The authors provide the first evidence that, even if Neanderthals predominantly consumed meat, they also had a remarkable plant intake, and they suggest the presence of a specific GM involved in cholesterol metabolism throughout human evolution. On the other hand, a glimpse of the ancestral human GM configuration could be provided by the GM of close primate relatives (Ley et al., 2008b; Moeller et al., 2012). Interestingly, the GM of modern humans clusters with that of other omnivorous primates, regardless of their affiliation to Pan (Ley et al., 2008b). This supports the key role of dietary habits in shaping the composition of the GIT microbial ecosystem.
The dynamics of the GM-host co-evolution and environmental adaptation can be addressed by investigating the GM variability in modern human populations of different culture (Candela et al., 2012). Indeed, the study of the GM from large healthy human populations of different age and socio-economic, geographic, and cultural settings allows researchers to point out the contribution of these environment components to the GM variation. In this context, a very recent paper explored the GM of the Hadza of Tanzania, a modern population of hunter-gatherers that still live as Paleolithic humans (Schnorr et al., 2014). This study elucidated the mechanisms of humans/GM co-evolution and showed a first map of the microbiota composition of the Hadza that reflects the functional adaptation to a foraging lifestyle. For instance, the high bacterial diversity and the enrichment in fibrolytic microorganisms (e.g., xylan-degrading Prevotella and Treponema) proper of the Hadza GM, represent ecosystem adaptations to provide SCFAs from their heavy plant-based diet. Furthermore, the Hadza show a sex-related divergence in the GM composition reflecting the sexual division of labor and sex differences in diet composition. In particular, the higher relative abundance of Treponema found in Hadza women could provide specific functions to deal with their higher intake of tubers and plant foods. In fact, women selectively forage for tubers and plant foods and spend a lot of time in camp, while men are highly mobile foragers and range far from the central camp site to obtain meat and honey. Even if foods are brought back to the camp and shared, men and women tend to consume more of their targeted foods. Finally, the absence of Bifidobacterium and a corresponding enrichment of potential opportunists as Proteobacteria and Spirochaetes in the Hadza GM probably correspond to a different tolerogenic layout of their immune system, redefining the notion of what we consider a healthy and an unhealthy GM structure. Indeed, the Hadza have relatively low rates of infectious diseases, metabolic diseases and nutritional deficiencies in comparison with other groups settled in Northern Tanzania (Bennett et al., 1973; Work et al., 1973; Blurton Jones et al., 1992). Moreover, De Filippo et al. (2010) compared the GM of children living in rural Africa and that of European children, and many differences emerged. Children from Boulpon Rural Village in Burkina Faso have a traditional rural African diet that is rich in starch, fibers, and plant polysaccharides and low in fat and animal protein, while European children follow a Western diet. The authors argued that the consumption of sugar, animal fat, and calorie-dense foods in industrialized countries is rapidly limiting the adaptive potential of the microbiota, by reducing microbiota richness and its functionality. Interestingly, the authors reported that only the GM of African children contains Prevotella, Xylanibacter, and Treponema that are involved in cellulose and xylan hydrolysis. It was speculated that the high fiber intake characterizing the African diet could change the GM composition to maximize the metabolic energy extraction from ingested plant polysaccharides (De Filippo et al., 2010). Finally, the same approach was applied to fecal samples from 531 children and adults from the Amazonas of Venezuela, rural Malawi and US metropolitan areas, including parents, siblings, and twins (Yatsunenko et al., 2012). The phylogenetic composition of the GM of these three populations is different, especially for US residents vs. non-US residents (Malawians and Amerindians). 
Furthermore, the authors confirmed the importance of Prevotella as a discriminatory taxon that distinguishes non-US from US individuals. A meta-analysis of the GM composition in Western populations (i.e., USA and Italian citizens), rural Malawi and Burkina Faso populations, and Hadza hunter-gatherers has been carried out (Schnorr et al., 2014). Data allowed to reconstruct the putative trajectory of GM adaptive evolution that accompanied human beings along the transition from the Paleolithic huntergatherer to the Neolithic rural communities until modern Western societies. The diagram reported in Figure 1 shows the emergence of specific co-abundance groups (CAGs) - groups of microorganisms which correlate and cluster together - along with the most important transition phases in our recent evolutionary history, such as the higher abundance of Ruminococcaceae unclassified CAG distinguishing for the Hadza hunter-gatherers, the emergence of Clostridiales unclassified and Prevotella CAGs in rural Malawi and Burkina Faso populations, and the dominance of the Faecalibacterium CAG in Western populations (Schnorr et al., 2014).

A striking and fascinating example for gene acquisition by a gut microbe as an adaptation to the local diet is described by Hehemann et al. (2010) in a study on Japanese population. By comparing the GM from Japanese and North American populations, it was reported that the GM of Japanese subjects is enriched for genes (probably acquired by contact with marine microbes) that encode enzymes capable of degrading porphyran

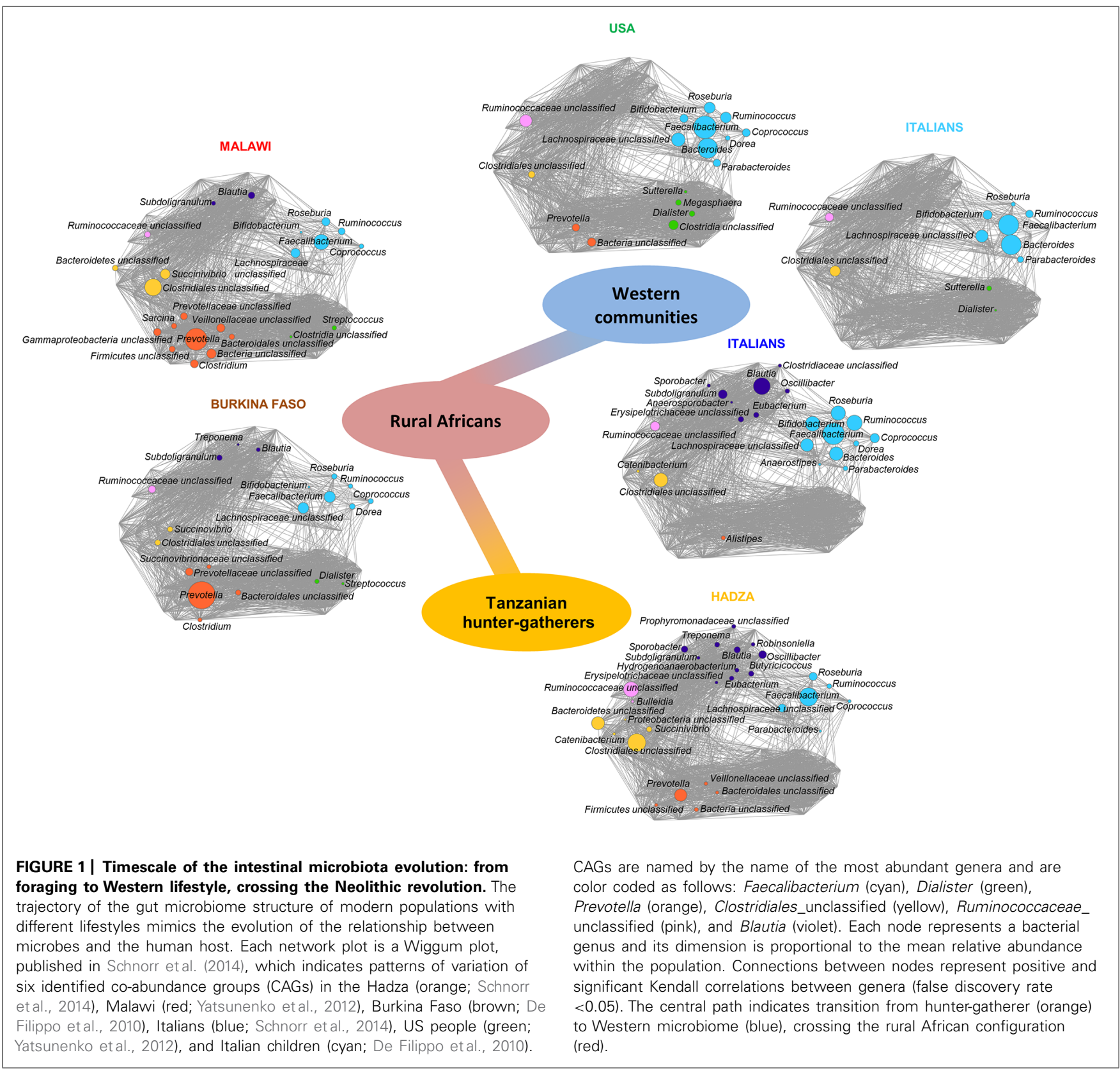


that is contained in seaweeds. In the Japanese culture, the greatest source of porphyran is due to nori (edible seaweed), which is commonly used in the preparation of sushi and it is the most produced and consumed seaweed for centuries in Japan.

\section{CONCLUSION}

Human beings faced tremendous changes in lifestyle and dietary habits along the course of their recent evolutionary history. They passed from Paleolithic hunter-gathering societies to Neolithic agricultural farmers until modern Westernized societies in 10,000 years, adapting to dramatic changes in diet and lifestyle in a relatively short evolutionary frame.

Human beings have been recently revised as superorganisms as a result of a close mutualistic relationship with their GM. Recent longitudinal studies highlighted an adaptive role for the GM in human biology, allowing to optimize the superorganism metabolic performances in response to diet, lifestyle, and physiological changes such as aging. This raises the question of whether this adaptive GM potential had a role in our recent evolutionary history, allowing adaptation to the profound lifestyle changes and describing a real GM-host evolutionary trajectory.

The first and very recent description of the Hadza GM structure provided important light in this direction. The peculiar structural and functional configuration of the Hadza gut microbial ecosystem suggests that adaptive functional changes of the GM accompanied the evolutionary trajectory of human beings, allowing the optimization of the superorganism performances in response to the profound changes that characterized our recent evolutionary history.

However, several circumstances characteristic of the Western world are challenging the resilience of the GM-host mutualistic interaction. The high-fat high-sugar energy-dense diet, sanitization, and antibiotic usage - a landmark of Western societies - are forcing GM adaptive changes to deviate from a mutualistic configuration. This raises the need to better comprehend the dynamics involved in this process, controlling any variables to preserve the extraordinary mutualistic relationship we evolved with our microbial counterpart.

\section{ACKNOWLEDGMENTS}

We thank and mention the Italian Ministry of University and Research (Project PRIN 2012 to Donata Luiselli), the European Union's Seventh Framework Programme (FP7/2007-2011) under grant agreement n. 259679 (IDEAL) and ICT-2011-9, n. 600803 (MISSION-T2D).

\section{REFERENCES}

Abrahamsson, T. R., Jakobsson, H. E., Andersson, A. F., Björkstén, B., Engstrand, L., and Jenmalm, M. C. (2013). Low gut microbiota diversity in early infancy precedes asthma at school age. Clin. Exp. Allergy 44, 842-850. doi: 10.1111/cea. 12253

Arpaia, N., Campbell, C., Fan, X., Dikiy, S., van der Veeken, J., deRoos, P., et al. (2013). Metabolites produced by commensal bacteria promote peripheral regulatory T-cell generation. Nature 504, 451-455. doi: 10.1038/nature 12726

Arumugam, M., Raes, J., Pelletier, E., Le Paslier, D., Yamada, T., Mende, D. R., et al. (2011). Enterotypes of the human gut microbiome. Nature 473, 174-180. doi: 10.1038 /nature09944
Bäckhed, F., Ding, H., Wang, T., Hooper, L. V., Koh, G. Y., Nagy, A. (2004). The gut microbiota as an environmental factor that regulates fat storage. Proc. Natl. Acad. Sci. U.S.A. 101, 15718-15723. doi: 10.1073/pnas.0407076101

Bennett, F. J., Barnicot, N. A., Woodburn, J. C., Pereira, M. S., and Henderson, B. E. (1973). Studies on viral, bacterial, rickettsial and treponemal diseases in the hadza of Tanzania and a note on injuries. Hum. Biol. 45, 243-272.

Benson, A. K., Kelly, S. A., Legge, R., Ma, F., Low, S. J., Kim, J., et al. (2010). Individuality in gut microbiota composition is a complex polygenic trait shaped by multiple environmental and host genetic factors. Proc. Natl. Acad. Sci. U.S.A. 107, 18933-18938. doi: 10.1073/pnas.1007028107

Biagi, E., Candela, M., Turroni, S., Garagnani, P., Franceschi, C., and Brigidi, P. (2013). Ageing and gut microbes: perspectives for health maintenance and longevity. Pharmacol. Res. 69, 11-20. doi: 10.1016/j.phrs.2012. 10.005

Biagi, E., Nylund, L., Candela, M., Ostan, R., Bucci, L., Pini, E., et al. (2010). Through ageing, and beyond: gut microbiota and inflammatory status in seniors and centenarians. PLOS ONE 5:e10667. doi: 10.1371/journal.pone. 0010667

Bisgaard, H., Li, N., Bonnelykke, K., Chawes, B. L., Skov, T., Paludan-Müller, G., et al. (2011). Reduced diversity of the intestinal microbiota during infancy is associated with increased risk of allergic disease at school age. J. Allergy Clin. Immunol. 128, 646-652.e1-5. doi: 10.1016/j.jaci.2011.04.060

Blurton Jones, N. G., Smith, L. C., O’Connell, J. F., Hawkes, K., and Kamuzora, C. L. (1992). Demography of the hadza, an increasing and high density population of savanna foragers. Am. J. Phys. Anthropol. 89, 159-181. doi: 10.1002/ajpa.1330890204

Candela, M., Biagi, E., Maccaferri, S., Turroni, S., and Brigidi, P. (2012). Intestinal microbiota is a plastic factor responding to environmental changes. Trends Microbiol. 20, 385-391. doi: 10.1016/j.tim.2012.05.003

Candela, M., Biagi, E., Turroni, S., Maccaferri, S., Figini, P., and Brigidi, P. (2013). Dynamic efficiency of the human intestinal microbiota. Crit. Rev. Microbiol. 1-7. doi: 10.3109/1040841X.2013.813900

Centanni, M., Turroni, S., Consolandi, C., Rampelli, S., Peano, C., Severgnini, M., et al. (2013). The enterocyte-associated intestinal microbiota of breast-fed infants and adults responds differently to a TNF-a-mediated pro-inflammatory stimulus. PLoS ONE 8:e81762. doi: 10.1371/journal.pone.0081762

Claesson, M. J., Cusack, S., O’Sullivan, O., Greene-Diniz, R., de Weerd, H., Flannery, E., et al. (2011). Composition, variability, and temporal stability of the intestinal microbiota of the elderly. Proc. Natl. Acad. Sci. U.S.A. 108(Suppl. 1), 4586-4591. doi: 10.1073/pnas.1000097107

Clemente, J. C., Ursell, L. K., Parfrey, L. W., and Knight, R. (2012). The impact of the gut microbiota on human health: an integrative view. Cell 148, 1258-1270. doi: 10.1016/j.cell.2012.01.035

Collins, S. M., Surette, M., and Bercik, P. (2012). The interplay between the intestinal microbiota and the brain. Nat. Rev. Microbiol. 10, 735-742. doi: $10.1038 /$ nrmicro2876

Costello, E. K., Lauber, C. L., Hamady, M., Fierer, N., Gordon, J. I., and Knight, R. (2009). Bacterial community variation in human body habitats across space and time. Science 326, 1694-1697. doi: 10.1126/science.1177486

Costello, E. K., Stagaman, K., Dethlefsen, L., Bohannan, B. J., and Relman, D. A. (2012). The application of ecological theory toward an understanding of the human microbiome. Science 336, 1255-1262. doi: 10.1126/science. 1224203

Cryan, J. F., and Dinan, T. G. (2012). Mind-altering microorganisms: the impact of the gut microbiota on brain and behaviour. Nat. Rev. Neurosci. 13, 701-712. doi: 10.1038/nrn3346

David, L. A., Maurice, C. F., Carmody, R. N., Gootenberg, D. B., Button, J. E., Wolfe, B. E., et al. (2014). Diet rapidly and reproducibly alters the human gut microbiome. Nature 505, 559-563. doi: 10.1038/nature 12820

De Filippo, C., Cavalieri, D., Di Paola, M., Ramazzotti, M., Poullet, J. B., Massart, S., et al. (2010). Impact of diet in shaping gut microbiota revealed by a comparative study in children from europe and rural Africa. Proc. Natl. Acad. Sci. U.S.A. 107, 14691-14696. doi: 10.1073/pnas.1005963107

De Vadder, F., Kovatcheva-Datchary, P., Goncalves, D., Vinera, J., Zitoun, C., Duchampt, A., et al. (2014). Microbiota-generated metabolites promote metabolic benefits via gut-brain neural circuits. Cell 156, 84-96. doi: 10.1016/j.cell.2013.12.016 
Diaz Heijtz, R., Wang, S., Anuar, F., Qian, Y., Björkholm, B., Samuelsson, A., et al. (2011). Normal gut microbiota modulates brain development and behavior. Proc. Natl. Acad. Sci. U.S.A. 108, 3047-3052. doi: 10.1073/pnas.1010529108

Dominguez-Bello, M. G., Costello, E. K., Contreras, M., Magris, M., Hidalgo, G., Fierer, N., et al. (2010). Delivery mode shapes the acquisition and structure of the initial microbiota across multiple body habitats in newborns. Proc. Natl. Acad. Sci. U.S.A. 107, 11971-11975. doi: 10.1073/pnas.1002601107

Drago, L., Toscano, M., Rodighiero, V., De Vecchi, E., and Mogna, G. (2012). Cultivable and pyrosequenced fecal microflora in centenarians and young subjects. J. Clin. Gastroenterol. 46(Suppl. 1), S81-S84. doi: 10.1097/MCG.0b013e3182 693982

Faith, J. J., Guruge, J. L., Charbonneau, M., Subramanian, S., Seedorf, H., Goodman, A. L., et al. (2013). The long-term stability of the human gut microbiota. Science 341:1237439. doi: 10.1126/science. 1237439

Foster, J. A., and McVey Neufeld, K.-A. (2013). Gut-brain axis: how the microbiome influences anxiety and depression. Trends Neurosci. 36, 305-312. doi 10.1016/j.tins.2013.01.005

Franceschi, C., Bonafe, M., Valensin, S., Olivieri, F., De Luca, M., Ottaviani, E., et al. (2000a). Inflamm-aging. an evolutionary perspective on immunosenescence. Ann. N. Y. Acad. Sci. 908, 244-54. doi: 10.1111/j.1749-6632.2000. tb06651.x

Franceschi, C., Valensin, S., Bonafe, M., Paolisso, G., Yashin, A. I., Monti, D., et al. (2000b). The network and the remodeling theories of aging: historical background and new perspectives. Exp. Gerontol. 35, 879-896. doi: 10.1016/ S0531-5565(00)00172-8

Frank, D. N., Robertson, C. E., Hamm, C. M., Kpadeh, Z., Zhang, T., Chen, H., et al. (2011). Disease phenotype and genotype are associated with shifts in intestinalassociated microbiota in inflammatory bowel diseases. Inflamm. Bowel Dis. 17 179-184. doi: 10.1002/ibd.21339

Gill, S. R., Pop, M., Deboy, R. T., Eckburg, P. B., Turnbaugh, P. J., Samuel, B. S., et al. (2006). Metagenomic analysis of the human distal gut microbiome. Science 312, 1355-1359. doi: 10.1126/science.1124234

Grignolio, A., Mishto, M., Faria, A. M., Garagnani, P., Franceschi, C., and Tieri, P. (2014). Towards a liquid self: how time, geography, and life experiences reshape the biological identity. Front. Immunol. 5:153. doi: 10.3389/fimmu.2014.00153

Hanage, W. P. (2014). Microbiology: microbiome science needs a healthy dose of scepticism. Nature 512, 247-248. doi: 10.1038/512247a

Hehemann, J.-H., Correc, G., Barbeyron, T., Helbert, W., Czjzek, M., and Michel, G. (2010). Transfer of carbohydrate-active enzymes from marine bacteria to japanese gut microbiota. Nature 464, 908-912. doi: 10.1038/nature08937

Jost, T., Lacroix, C., Braegger, C. P., and Chassard, C. (2012). New insights in gut microbiota establishment in healthy breast fed neonates. PLoS ONE 7:e44595. doi: 10.1371/journal.pone.0044595

Kamada, N., Seo, S.-U., Chen, G. Y., and Núñez, G. (2013). Role of the gut microbiota in immunity and inflammatory disease. Nat. Rev. Immunol. 13, 321-335. doi: $10.1038 /$ nri3430

Kashyap, P. C., Marcobal, A., Ursell, L. K., Smits, S. A., Sonnenburg, E. D., Costello, E. K., et al. (2013). Genetically dictated change in host mucus carbohydrate landscape exerts a diet-dependent effect on the gut microbiota. Proc. Natl. Acad. Sci. U.S.A. 110, 17059-17064. doi: 10.1073/pnas.1306070110

Khachatryan, Z. A., Ktsoyan, Z. A., Manukyan, G. P., Kelly, D., Ghazaryan, K. A., and Aminov, R. I. (2008). Predominant role of host genetics in controlling the composition of gut microbiota. PLoS ONE 3:e3064. doi: 10.1371/journal.pone. 0003064

Koenig, J. E., Spor, A., Scalfone, N., Fricker, A. D., Stombaugh, J., Knight, R., et al. (2011). Succession of microbial consortia in the developing infant gut microbiome. Proc. Natl. Acad. Sci. 108(Suppl. 1), 4578-4585. doi: 10.1073/pnas 1000081107

Koren, O., Goodrich, J. K., Cullender, T. C., Spor, A., Laitinen, K., Bäckhed, H. K., et al. (2012). Host remodeling of the gut microbiome and metabolic changes during pregnancy. Cell 150, 470-480. doi: 10.1016/j.cell.2012. 07.008

Koropatkin, N. M., Cameron, E. A., and Martens, E. C. (2012). How glycan metabolism shapes the human gut microbiota. Nat. Rev. Microbiol. 10, 323-335. doi: 10.1038/nrmicro2746

Kovacs, A., Ben-Jacob, N., Tayem, H., Halperin, E., Iraqi, F. A., and Gophna, U. (2011). Genotype is a stronger determinant than sex of the mouse gut microbiota. Microb. Ecol. 61, 423-428. doi: 10.1007/s00248-010-9787-2
Ley, R. E., Lozupone, C. A., Hamady, M., Knight, R., and Gordon, J. I. (2008a) Worlds within worlds: evolution of the vertebrate gut microbiota. Nat. Rev Microbiol. 6, 776-788. doi: 10.1038/nrmicro1978

Ley, R. E., Hamady, M., Lozupone, C., Turnbaugh, P. J., Ramey, R. R., Bircher, J. S., et al. (2008b). Evolution of mammals and their gut microbes. Science 320, 1647-1651. doi: 10.1126/science.1155725

Lozupone, C. A., Stombaugh, J. I., Gordon, J. I., Jansson, J. K., and Knight, R. (2012). Diversity, stability and resilience of the human gut microbiota. Nature 489, 220-230. doi: 10.1038/nature11550

Maynard, C. L., Elson, C. O., Hatton, R. D., and Weaver, C. T. (2012). Reciprocal interactions of the intestinal microbiota and immune system. Nature 489, 231241. doi: 10.1038/nature11551

Moeller, A. H., Degnan, P. H., Pusey, A. E., Wilson, M. L., Hahn, B. H., and Ochman, H. (2012). Chimpanzees and humans harbour compositionally similar gut enterotypes. Nat. Commun. 3:1179. doi: 10.1038/ ncomms2159

Muegge, B. D., Kuczynski, J., Knights, D., Clemente, J. C., González, A., Fontana, L., et al. (2011). Diet drives convergence in gut microbiome functions across mammalian phylogeny and within humans. Science 332, 970-974. doi: 10.1126/science.1198719

Nicholson, J. K., Holmes, E., Kinross, J., Burcelin, R., Gibson, G., Jia, W., et al. (2012). Host-gut microbiota metabolic interactions. Science 336, 1262-1267. doi: $10.1126 /$ science. 1223813

O'Hara, A. M., and Shanahan, F. (2006). The gut flora as a forgotten organ. EMBO Rep. 7, 688-693. doi: 10.1038/sj.embor.7400731

Ottaviani, E., Ventura, N., Mandrioli, M., Candela, M., Franchini, A., and Franceschi, C. (2011). Gut microbiota as a candidate for lifespan extension: an ecological/evolutionary perspective targeted on living organisms as metaorganisms. Biogerontology 12, 599-609. doi: 10.1007/s10522-011-9352-5

Palmer, C., Bik, E. M., DiGiulio, D. B., Relman, D. A., and Brown, P. O. (2007). Development of the human infant intestinal microbiota. PLoS Biol. 5:e177. doi: 10.1371/journal.pbio.0050177

Petersson, J., Schreiber, O., Hansson, G. C., Gendler, S. J., Velcich, A., Lundberg, J. O., et al. (2011). Importance and regulation of the colonic mucus barrier in a mouse model of colitis. Am. J. Physiol. Gastrointest. Liver Physiol. 300, G327-G333. doi: 10.1152/ajpgi.00422.2010

Petnicki-Ocwieja, T., Hrncir, T., Liu, Y.-J., Biswas, A., Hudcovic, T., TlaskalovaHogenova, H., et al. (2009). Nod2 is required for the regulation of commensal microbiota in the intestine. Proc. Natl. Acad. Sci. U.S.A. 106, 15813-15818. doi: 10.1073/pnas.0907722106

Qin, J., Li, R., Raes, J., Arumugam, M., Burgdorf, K. S., Manichanh, C., et al. (2010). A human gut microbial gene catalogue established by metagenomic sequencing. Nature 464, 59-65. doi: 10.1038/nature08821

Rampelli, S., Candela, M., Turroni, S., Biagi, E., Collino, S., Franceschi, C., et al. (2013). Functional metagenomic profiling of intestinal microbiome in extreme ageing. Aging (Albany, NY) 5, 902-912.

Rautava, S., Luoto, R., Salminen, S., and Isolauri, E. (2012). Microbial contact during pregnancy, intestinal colonization and human disease. Nat. Rev. Gastroenterol. Hepatol. 9, 565-576. doi: 10.1038/nrgastro.2012.144

Ridaura, V. K., Faith, J. J., Rey, F. E., Cheng, J., Duncan, A. E., Kau, A. L., et al. (2013). Gut microbiota from twins discordant for obesity modulate metabolism in mice. Science 341:1241214. doi: 10.1126/science.1241214

Round, J. L., and Mazmanian, S. K. (2009). The gut microbiota shapes intestinal immune responses during health and disease. Nat. Rev. Immunol. 9, 313-323. doi: $10.1038 /$ nri2515

Russell, W. R., Hoyles, L., Flint, H. J., and Dumas, M.-E. (2013). Colonic bacterial metabolites and human health. Curr. Opin. Microbiol. 16, 246-254. doi: 10.1016/j.mib.2013.07.002

Schnorr, S. L., Candela, M., Rampelli, S., Centanni, M., Consolandi, C., Basaglia, G., et al. (2014). Gut microbiome of the hadza hunter-gatherers. Nat. Commun. 5:3654. doi: $10.1038 /$ ncomms4654

Schulz, M. D., Atay, C., Heringer, J., Romrig, F. K., Schwitalla, S., Aydin, B., et al. (2014). High-fat-diet-mediated dysbiosis promotes intestinal carcinogenesis independently of obesity. Nature 514, 508-512. doi: 10.1038/nature13398

Segain, J. P., Raingeard de la Blétière, D., Bourreille, A., Leray, V., Gervois, N., Rosales, C., etal. (2000). Butyrate inhibits inflammatory responses through NFkappaB inhibition: implications for Crohn's Disease. Gut 47, 397-403. doi: 10.1136/gut.47.3.397 
Sistiaga, A., Mallol, C., Galván, B., and Summons, R. E. (2014). The eanderthal meal: a new perspective using faecal biomarkers. PLoS ONE 9:e101045. doi: 10.1371/journal.pone.0101045

Stewart, J. A., Chadwick, V. S., and Murray, A. (2005). Investigations into the influence of host genetics on the predominant eubacteria in the faecal microflora of children. J. Med. Microbiol. 54, 1239-1242. doi: 10.1099/jmm.0.46189-0

Sudo, N., Chida, Y., Aiba, Y., Sonoda, J., Oyama, N., Yu, X.-N., et al. (2004). Postnatal microbial colonization programs the hypothalamic-pituitaryadrenal system for stress response in mice. J. Physiol. 558, 263-275. doi: 10.1113/jphysiol.2004.063388

Trompette, A., Gollwitzer, E. S., Yadava, K., Sichelstiel, A. K., Sprenger, N., Ngom-Bru, C., et al. (2014). Gut microbiota metabolism of dietary fiber influences allergic airway disease and hematopoiesis. Nat. Med. 20, 159-166. doi: 10.1038/nm.3444

Turnbaugh, P. J., Hamady, M., Yatsunenko, T., Cantarel, B. L., Duncan, A., Ley, R. E., et al. (2009). A core gut microbiome in obese and lean twins. Nature 457, 480-484. doi: $10.1038 /$ nature 07540

Turnbaugh, P. J., Ley, R. E., Hamady, M., Fraser-Liggett, C. M., Knight, R., and Gordon, J. I. (2007). The human microbiome project. Nature 449, 804-810. doi: 10.1038 /nature06244

Vaahtovuo, J., Toivanen, P., and Eerola, E. (2003). Bacterial composition of murine fecal microflora is indigenous and genetically guided. FEMS Microbiol. Ecol. 44, 131-136. doi: 10.1016/S0168-6496(02)00460-9

Vael, C., and Desager, K. (2009). The importance of the development of the intestinal microbiota in infancy. [Miscellaneous Article]. Curr. Opin. Pediatrics 21, 794-800. doi: 10.1097/MOP.0b013e328332351b

Vanhoutte, T., Huys, G., Brandt, E., and Swings, J. (2004). Temporal stability analysis of the microbiota in human feces by denaturing gradient gel electrophoresis using universal and group-specific 16S rRNA gene primers. FEMS Microbiol. Ecol. 48 , 437-446. doi: 10.1016/j.femsec.2004.03.001

Wacklin, P., Tuimala, J., Nikkilä, J., Tims, S., Mäkivuokko, H., Alakulppi, N., et al. (2014). Faecal microbiota composition in adults is associated with the FUT2 gene determining the secretor status. PLoS ONE 9:e94863. doi: 10.1371/journal.pone.0094863

Walker, A. W., Ince, J., Duncan, S. H., Webster, L. M., Holtrop, G., Ze, X., et al. (2011). Dominant and diet-responsive groups of bacteria within the human colonic microbiota. ISME J. 5, 220-230. doi: 10.1038/ismej.2010.118
Walter, J., and Ley, R. (2011). The human gut microbiome: ecology and recent evolutionary changes. Ann. Rev. Microbiol. 65, 411-429. doi: 10.1146/annurevmicro-090110-102830

Work, T. H., Ifekwunigwe, A., Jelliffe, D. B., Jelliffe, P., and Neumann, C. G. (1973). Tropical problems in nutrition. Ann. Intern. Med. 79, 701-711. doi: 10.7326/00034819-79-5-701

Wu, G. D., Chen, J., Hoffmann, C., Bittinger, K., Chen, Y.-Y., Keilbaugh, S. A., et al. (2011). Linking long-term dietary patterns with gut microbial enterotypes. Science 334, 105-108. doi: 10.1126/science.1208344

Yatsunenko, T., Rey, F. E., Manary, M. J., Trehan, I., Dominguez-Bello, M. G., Contreras, M., etal. (2012). Human gut microbiome viewed across age and geography. Nature 486, 222-227. doi: 10.1038/nature 11053

Zhang, C., Zhang, M., Wang, S., Han, R., Cao, Y., Hua, W., et al. (2010) Interactions between gut microbiota, host genetics and diet relevant to development of metabolic syndromes in mice. ISME J. 4. 232-241. doi: 10.1038/ismej. 2009.112

Conflict of Interest Statement: The authors declare that the research was conducted in the absence of any commercial or financial relationships that could be construed as a potential conflict of interest.

Received: 18 April 2014; paper pending published: 30 April 2014; accepted: 17 October 2014; published online: 04 November 2014.

Citation: Quercia S, Candela M, Giuliani C, Turroni S, Luiselli D, Rampelli S, Brigidi P, Franceschi C, Bacalini MG, Garagnani P and Pirazzini C (2014) From lifetime to evolution: timescales of human gut microbiota adaptation. Front. Microbiol. 5:587. doi: $10.3389 /$ fmicb.2014.00587

This article was submitted to Evolutionary and Genomic Microbiology, a section of the journal Frontiers in Microbiology.

Copyright (C) 2014 Quercia, Candela, Giuliani, Turroni, Luiselli, Rampelli, Brigidi, Franceschi, Bacalini, Garagnani and Pirazzini. This is an open-access article distributed under the terms of the Creative Commons Attribution License (CC BY). The use, distribution or reproduction in other forums is permitted, provided the original author(s) or licensor are credited and that the original publication in this journal is cited, in accordance with accepted academic practice. No use, distribution or reproduction is permitted which does not comply with these terms. 